\title{
Simulation of motor-neuronal networks
}

\author{
MICHAEL YOST, FREDERICK BREMNER, and ROBERT STRAUSS \\ Trinity University, San Antonio, Texas
}

\begin{abstract}
This research develops a theoretical connectionist-type model of the operation of the human motor system. The model includes the operation of the cerebral cortex, pons, spinal cord, and muscles. It emphasizes the parallel passage of neuronal signals, the integration of multiple sig. nals within nuclei, and the modulation of signals as a result of sensory input. A redundant Fast Fourier Transform (FFT) procedure that analyzes EEG signals by a method similar to the one used by pontine nuclei is described.
\end{abstract}

For this work, we assume that the human brain processes data in parallel and that the motor system is organized both hierarchically and gubernatorially (Gallistel, 1980). By hierarchically we mean that a signal to initiate a planned motor act proceeds from the motor cortex of the brain to the pons, to the spinal cord, and then to the muscles. By gubernatorially we mean that the pons and spinal cord have considerable responsibility for modulating or updating the motor signals that pass through them.

If we follow the work of Gallistel (1980), the algorithm of the cortex of the brain is a Fourier analysis of all possible vectors to move from Point $A$ to Point $B$. The output of the motor cortex to the lower motor centers is a "sinusoidally oscillating signal whose period, amplitude, and phase specify the period, amplitude, and phase of the desired trajectory..." (Gallistel, 1980, p. 369) of the motor movement to be performed. In addition to this sinusoidally oscillating signal, the cortex sends the horizontal, vertical, and distance Cartesian coordinates of the physical movement to be made. Therefore, Gallistel represents the motor engram as a vector containing six elements: period, amplitude, phase, and the three directional Cartesian coordinates. As the vector is sent from the motor cortex to the pons, information from the cerebellum and other position sensors are used to modulate it. The modulated vector is passed from the pons to the spinal cord, where it is again modulated. The information used to modulate the vector at the level of the spinal cord is obtained from the muscle spindles. As a motor task is being completed, the pons and the spinal cord each continue to modulate the vector, which carries the signal between the motor cortex of the brain and the muscles in an independent, iterative process (i.e., parallel process) until the motor task is completed. Since Hinton and Anderson (1981) estimated that the brain can make a limited number of serial iterations per unit time (approximately 100 per $250 \mathrm{msec}$ ), we know that this iterative process is finite, and not infinite, in nature.

Please address all correspondence to Michael Yost, Office of the President, Trinity University, San Antonio, TX 78284.

\section{RATIONALE}

Figure 1 is a simplistic neuroanatomical model containing the components required for a simple motor task. The signals (vectors) to initiate and complete the motor task originate in the motor cortex of the brain and travel along parallel paths through neurons (lettered A through D) to an individual nucleus in the pons. The individual signals or vectors traveling through these neurons are integrated into a complex signal in the nucleus. Information obtained from sensory organs about the stage of completion of the task is used by the nucleus to modulate or modify the complex signal as it is sent (along Neurons E through G) to the spinal cord. There is no reason to believe that the neurons labeled $E, F$, and $G$ are continuations of Neurons A, B, C, and D or that equal numbers of neurons lead into and out of a nucleus in the pons. In fact, because neurons are polysynaptic, there are probably fewer axons dealing with the original signal on the output side of a nucleus in the pons than on the input side. As the signal passes through the spinal cord, information obtained from the muscle spindles is used to moderate the signal as it is sent to the muscles.

The hierarchical nature of this model is obvious. The signal to perform a motor task begins at one level within the brain and is transmitted in serial sequence from level to level until it reaches the muscles and initiates the motor activity. The gubernatorial characteristic of the model is in operation at two levels. Both the nuclei in the pons and the spinal cord receive information that they use to moderate the signal they receive, integrate, and transmit to the next level of the model. Moreover, this gubernatorial characteristic is sequential in nature. That is, the signal that is first moderated by the pons is again moderated by the spinal cord. This constitutes a type of "fine tuning" of the signal as it passes from level to level within the model. Also, a motor task is not completed with a single signal but a series of signals sent along parallel neurons that are iteratively refined as the task is completed. 


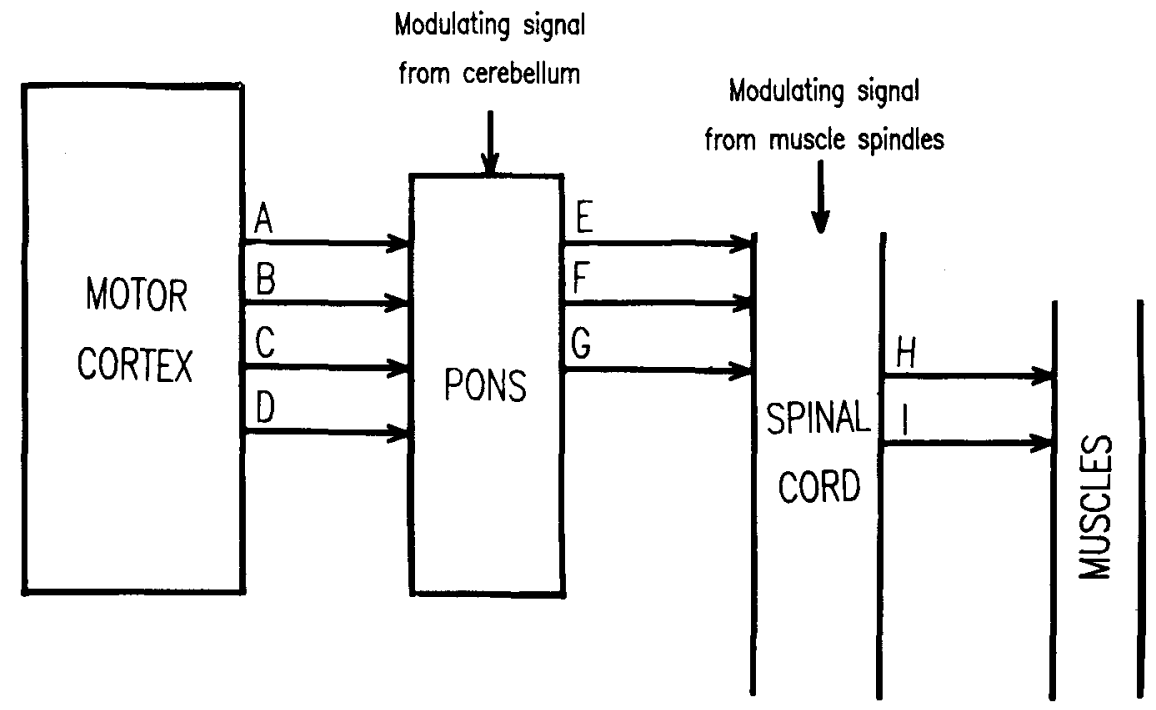

Figure 1. A simplistic neuroanatomical model containing the components required for a simple motor task. See text for details.

\section{THE MODEL}

Several studies have investigated the Cartesian components of a Gallistel-type model (Llinas \& Pellionisz, 1985; Pellionisz, 1983; Pellionisz \& Llinas, 1980). In the current research, our attention is directed to the sinusoidally oscillating component of the Gallistel (1980) model.

Let us assume that the firing of multiple neurons in the motor cortex sends analog-"type" signals (action potentials) down many parallel lines (axons) to the next lower level of the motor system. In our model, the pons is the next lower level of this system. The function of the pons is to monitor, integrate, and modulate the cortical message. We propose that the nuclei in the pons view the signals of the individual cortical neurons as a complex signal. That is, it is the pattern of signals as seen across a set of parallel lines that is the motor engram. This complex signal is interpreted and modulated within the nuclei of the pons and sent through yet other neurons to the spinal cord. The nuclei of the pons send a series of action potentials down parallel lines that are analog- "type" signals to the spinal cord, where the process is repeated before the signal is finally sent to the muscles. The spinal cord then does two things: (1) it sends a message to initiate action in the muscles, and (2) it sends a message back to the pons. Both of these output messages are a series of action potentials traveling down parallel lines.

Our model focuses on the process carried out by the nuclei of the pons in interpreting the signals that they receive from the motor cortex. If, as in Figure 1, signals received from individual cortical neurons are combined in the nuclei of the pons, then the integrated signal should be an approximation of the type of signal collected in EEG research. That is, the integrated signal in a pontine nucleus is an approximation of the algebraic sum of the individual signals that are input from the cortical neurons. If this is true, then the nuclei of the pons (and those in other areas of the brain as well) must be performing the equivalent of an almost continuous Fast Fourier Transform (FFT) of the signals that they receive.

In the laboratory, we use FFTs of successive 1-sec samples of data to draw inferences about brain activity. This is a time-consuming task, even with a high-speed computer. Given human reaction times and the rate at which neurons can fire and recycle, the multiple nuclei of the pons must be performing FFTs at a much higher rate and with a more efficient sampling technique than we do in the laboratory. It has been hypothesized (Ballard \& Brown, 1985; Feldman, 1985) that the reason the brain is so much faster at information processing than are digital computers is that the nuclei of the brain process information in parallel. The computer industry is now developing a computer to match the brain's method of parallel processing (Elmer-Dewitt, 1986). This new computer is called the Connection Machine and performs calculations for equations like the FFT simultaneously in different locations in the computer rather than serially in a single location, as our laboratory computer does. Although no one really knows how fast the neurons in the pons can parallel process the incoming signals, we believe that, given standard reaction times of $125 \mathrm{msec}$, there is sufficient time for the necessary calculations to be completed.

\section{THE SIMULATION}

We have developed a simulation of an approximation of the FFT process that takes place in the nuclei. In this simulation, the Fourier analysis is conducted at each level of the motor system independently of the other levels of the system but overlapping in time with all other levels of the system. That is, the output of each nuclear level is a series of action potentials traveling down a set of 
parallel lines that appear to the next lower level as a sinusoidal signal. We call the decoding process performed by the next lower level on this complex parallel signal a redundant FFT (RFFT).

It is the sampling and analysis rate that makes the RFFT different from the more traditional FFT. Figure 2 is a representation of digitized data gathered from a single electrode on a subject for a series of $T$ seconds. At approximately $T / 2$ seconds, the subject began to respond to a new stimulus, which caused the change in the EEG pattern. If a traditional approach were used, we would divide the data into 1-sec units before and after the delivery of the stimulus, calculate the FFTs of these 1-sec data segments, and compare the results of the FFTs to determine whether the stimulus had an effect. Using a RFFT, we begin by taking the data for the 1st second (Segment A in Figure 2) and perform a FFT. Once the data for the 1st second are analyzed, the simulation program moves $1 / 4 \sec$ (64 data points, assuming an A/D sampling rate of 256 per second) down the data, takes another 1-sec sample of data, and performs the next FFT. The second and third data segments are represented as $B$ and $C$ in Figure 2. This analysis procedure is like taking a $1-\mathrm{sec}$ window that an investigator lays on and slides along the vector of data analyzing each successive, overlapping second looking for places where there are changes in the signal. This process of taking repeated 1-sec samples of data that are $1 / 4$ sec apart continues until the end of the data is reached. Each successive 1-sec sample of data is made up of three fourths of the signal from the previous sample and one fourth of the signal from the next section of the data. The RFFT gives a continuous view of a continuous data signal and is sensitive to and easily identifies places in the data where changes occur. The mathematical equations and algorithm used in the FFT portion of the simulation were taken from the International Mathematics Statistics Library (1984).

For explanation purposes, the RFFT simulation was described above as taking 1-sec samples at $1 / 4-\mathrm{sec}$ intervals; in fact, however, the step rate in the simulation program is variable and parameter driven. The simulation program produces both the power and percent power spectra for $1-\mathrm{Hz}$ windows between 2 and $13 \mathrm{~Hz}$. When the program is set to analyze $1-\mathrm{sec}$ units of data at $1 / 4-\mathrm{sec}$ intervals, the program produces four spectra for each second of data except for the last second. For $10 \mathrm{sec}$ of data, the program produces 37 spectra.

The RFFT is a statistical technique that we believe analyzes data using basically the same approach as do the nuclei in the pons. The model is based on the assumption that the nuclei in the pons integrate the signals that they are sent and constantly evaluate the signals as the signals are received (conducting RFFTs). When the signal that a nucleus receives is relatively constant or nonchanging over time, then the signal that it sends to the spinal cord is also relatively constant and nonchanging. Conversely, when a nucleus analyzes (RFFTs) the incoming signal and notes a change, it makes the appropriate changes in the signal that it sends to the spinal cord.

In the traditional use of a FFT, an investigator analyzes sequential, nonoverlapping 1-sec units of data. There are an almost infinite number of neurons in the brain, and

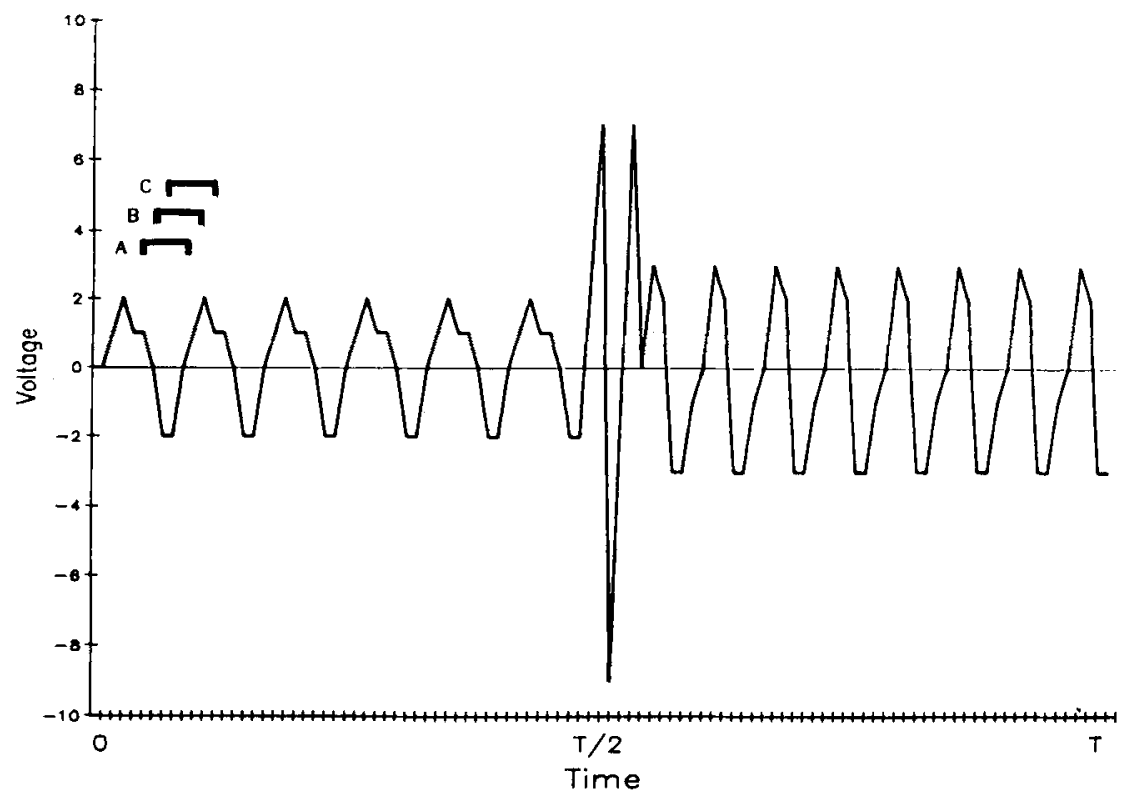

Figure 2. A representation of digitized data gathered from a single electrode on a subject for a series of $T$ seconds. See text for details. 
these neurons can fire as rapidly as every $1,000 \mathrm{msec}$. The large standard deviations associated with the power spectra of data that have undergone a traditional FFT are evidence of the rapid rate of change of neuron activity in the brain (Yost, Bremner, \& Fox, 1985). The traditional use of the FFT does not approximate the brain that it is designed to evaluate and describe, and we believe that it does not give a complete measure of the brain's activity.

The RFFT developed as a part of this research is designed to measure and describe the neuronal activity at given locations in the brain on the basis of an algorithm that we believe approximates the neuronal function itself. This is a statistical technique that produces information from which an investigator can draw inferences from descriptive data about brain activity in a subject; it is not a technique designed to produce data that can be used with inferential statistics. Although the stepwise approach used to analyze the data with the RFFT uses an overlapping data-sampling technique, this is not a moving-average approach to the analysis of data.

To give an example of the use and interpretation of the RFFT, let us assume a single-subject (human), single-trial study in which data are gathered from a single electrode located in a pontine nucleus. At the beginning of the session, the subject is seated; after $10 \mathrm{sec}$ of baseline data are gathered, the subject is instructed to "stand up." Ten seconds of standing-up data are then gathered. If we assume that 1 -sec units of data at $1 / 4-\sec$ steps are analyzed, then a 12 (one for each of the $1-\mathrm{Hz}$ power spectra windows) $\times 75$ (four for each of the first $19 \mathrm{sec}$ and one for the last second) matrix is developed. The data can be interpreted from the matrix, but interpretation is made easier if the data are plotted as a line graph. Since the data sampling in a RFFT uses an overlapping technique, the rate of change in the power from sample to sample within a stimulus condition (down any one of the columns) is relatively small. However, when the $1-\mathrm{Hz}$ sampling window is moved along the data and encounters the new stimulus condition (at the 10-sec point in the data), the power in each of the $1-\mathrm{Hz}$ windows begins to shift rapidly and later becomes stable under the new stimulus condition. These data are consistent with the operation of our proposed model. That is, the nuclei of the pons continuously monitor the data that they receive, evaluate that data, and respond to the changes by sending the appropriate signals to the next level of the motor system.

\section{DISCUSSION}

If one follows the connectionist model of logic (Feldman, 1985; Feldman \& Ballard, 1982; Hinton \& Anderson, 1981), in which parallel axons spatially sum on higher order neurons, these neurons either individually or collectively (nucleus) must decode the spatially aggregated signal. The combined or integrated signal is more analog than binary in nature (Gallistel, 1980), yet it is just this combined signal that the connectionists believe is the en- gram. Therefore, we believe that these neurons or nuclei must continually monitor and discriminate any change in this analog signal and that this monitoring and discriminating process is well simulated by the RFFT.

Animal lesion studies (Polit \& Bizzi, 1978) support the concept that afferent feedback adaptively modifies learned motor programs. As early as 1973, Evarts reported that lower neural centers in the motor systems had strong gubernatorial powers. More recently, Berkthoz and Melville-Jones (1985), after reviewing the current oculomotor research literature, were willing to venture that behavioral influences modified even the simplest reflexes. Although it is important for our parallel-processing model that simple reflexes be modifiable, it is the distributedprocessing aspect of the motor-neuronal network that is being simulated. In this sense, our model is similar to that of Pellionisz and his co-workers (Llinas \& Pellionisz, 1985; Pellionisz, 1983; Pellionisz \& Llinas, 1980), who presented the sensory-motor signal-processing system as a series of mathematical transformations molding the neural message at every level. There seems to be consensus that the motor system is hierarchically organized and that less responsibility is given to the cortex than classical theory would suggest. In particular, lower motor neurons are free to alter such parameters as speed of arm movement (Kelle \& Posner, 1968). In the simulation presented here, we believe that the message to modify a movement by lower brain centers is arrived at by a RFFT-like process.

\section{REFERENCES}

BAllaRd, D. A., \& Brown, C. M. (1985). Vision. BYTE, 10, 241-261. Berkthoz, A., Melville-Jones, G. (1985). Review of oculomotor research: 1 . Amsterdam: Elsevier.

ElMER-DEWitT, P. (1986). Letting 1000 flowers grow. Time, 127, 64.

EvarTs, E. V. (1973). Brain mechanisms in movements. Scientific American, 299, 96-103.

Feldman, J. A. (1985). Connectionists. BYTE, 10, 265-273.

Feldman, J. A., \& Ballard, D. H. (1982). Connectionist models and their properties. Cognitive Science, 6, 205-245.

Gallistel, C. R. (1980). The organization of action. Hillsdale, NJ: Erlbaum.

Hinton, G. E., \& Anderson, J. A. (1981). Parallel models of associative memory. Hillsdale, NJ: Erlbaum.

International Mathematics Statistical Library. (1984). Houston, TX. Kelle, S. W., Posner, M. I. (1968). Processing of visual feedback in abstract ideas. Joumal of Experimental Psychology, 77, 155-158.

Llinas, R., Pellonisz, A. (1985). Cerebellar function and adaptive features in the central nervous system. In A. Berkthoz \& G. Melville-Jones (Eds.), Reviews of oculomotor research: I (pp. 281296). Amsterdam: Elsevier.

Pellonisz, A. (1983). Brain theory: Connecting neurobiology to robotics. Journal of Theoretical Biology, 110, 333-335.

Pellionisz, A., \& LuNAs, R. (1980). Tensoral representation of spacetime. In CNS: Sensory-Motor Coordination via Distributed Cerebellar Space-time Metric. Journal of Society of Neuroscience, 6, 510.

Polrt, A., \& BizzI, E. (1978). Processes controlling arm movement in monkeys. Science, 231, 1292-1293.

Yost, M., Bremner, F., Fox, C. (1985, April). Biofeedback: A longitudinal study on a single subject. Paper presented at the meeting of the Biofeedback Society of America, New Orleans, LA. 